International Journal of Engineering \& Technology, 7 (3.29)(2018) 150-152
International Journal of Engineering \& Technology
SPC
Website: $w$ ww.sciencepubco.com/index.php/IJET
Research paper

\title{
Theory of a quantum artificial neuron based on superconducting devices
}

\author{
Haruna Katayama $^{1}$, Toshiyuki Fujii ${ }^{2}$, Noriyuki Hatakenaka ${ }^{3}$ \\ ${ }^{1}$ Faculty of Integrated Arts and Sciences, Hiroshima University, 1-7-1 Kagamiyama, Higashi-Hiroshima, Japan \\ ${ }^{2}$ Department of Physics, Asahikawa Medical University, 2-1-1-1 Midorigaoka-higashi, Asahikawa, Japan \\ ${ }^{3}$ Graduate School of Integrated Arts and Sciences, Hiroshima University, 1-7-1 Kagamiyama, Higashi-Hiroshima, Japan \\ *Corresponding author E-mail: halna496@gmail.com
}

\begin{abstract}
An artificial neuron using superconducting devices, so-called rf SQUID, working at the quantum-mechanical domain is studied. It is shown that quantum rf SQUID regarded as flux qubit can act as an artificial neuron with sigmoid function generated by coherent quantummechanical transitions between wells in double well potential representing rf SQUID.
\end{abstract}

Keywords: Artificial Neural Networks; Superconducting Quantum Interference Devices (Squid); Sigmoid Function; Superconducting Neurons.

\section{Introduction}

Artificial Intelligence has emerged as a practical technology, with successful applications in many fields like pattern recognition, especially when the underlying data relationship is unknown. An artificial neural network (ANN) inspired by biological nervous networks is a key technology to support artificial intelligence [1]. Basic building block of ANN is an artificial neuron with three simple sets of rules: multiplication, summation and activation. The typical ANN consists of huge number of interconnected such artificial neurons, which are stacked sequentially in rows that are known as layers as shown in Fig. 1 (a). An artificial neuron receives signals from other neurons through synapses located on the dendrites of the neuron and combines them and applies a nonlinear operation to the combined signal, in order to judge whether to activate the neuron for signaling the neurons in the subsequent layer. Therefore, the activation function is a heart of the artificial neuron.

So far, several activation functions suitable for each task have been considered. Among them, sigmoid or logistic functions are most effective for ANN on which the error backpropagation learning algorism is implemented [2] because of their simple mathematical handling, especially their differentiation.

The hardware implementation of sigmoid functions has been initially investigated using semiconductor integrated circuits. Later, superconducting circuits are considered to be an alternative candidate to overcome difficulties in semiconductors such as large power dissipation. In fact,

ANN using superconducting circuits with their ultra-high-speed operation, ultra-low-power consumption and scalability enabled by nanotechnology has successfully been implemented in various ways [4]-[10]. However, the physical basis for generating the sigmoid function was not sufficient. In our previous paper [11], we clarified the physical basis for sigmoid function generation and applied it to artificial neurons based on superconducting quantum interference devices (SQUIDs).
Recent advances in nanotechnology have led the system size to nanoscale, where devices that have been operating on the principle of classical mechanics have to be dealt with quantum-mechanically.

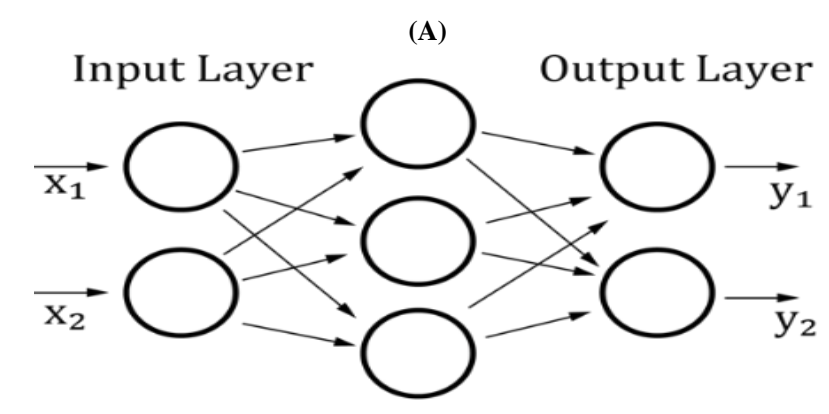

Hidden Layer

(B)

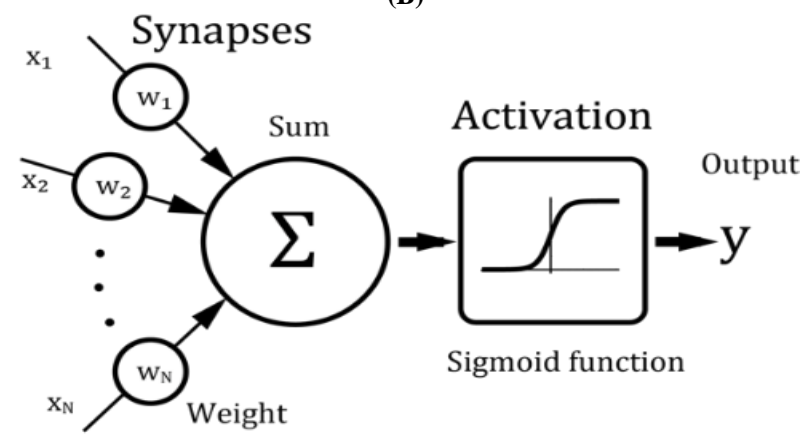

Inputs

Fig. 1: Schematic Diagrams of (A) A Typical Three-Layered Feed-Forward Artificial Neural Network and (B) an Artificial Neuron.

In this paper, we reconsider the generation mechanism of the sigmoid function required for artificial neurons based on the principle 
of quantum mechanics and analyze SQUID-based artificial neurons which operate in quantum-mechanical domain.

\section{Classical squid-based artificial neurons}

Here we briefly review classical artificial neurons based on a radio frequency (rf) SQUID discussed in the previous paper [11] for the preparation to develop into neurons operating in quantum mechanical domain below.

a) Physical origin of sigmoid function generation

We showed that the physical origin of the sigmoid function generation was the transition processes between two states in double well potential. The probability of finding a particle in one of the two wells, $p$, in thermal equilibrium is derived from the rate equation on the transition between two states in the double well potential as shown in Fig. 2 as follows;

$$
\mathrm{p}=\frac{1}{1+\mathrm{e}^{-\Delta \mathrm{E} / \mathrm{kT}}}
$$

Where $\Delta \mathrm{E}$ is the energy difference between two states. $\mathrm{K}$ and $\mathrm{T}$ are the Boltzmann constant and temperature, respectively. This is the sigmoid function required for artificial neurons.

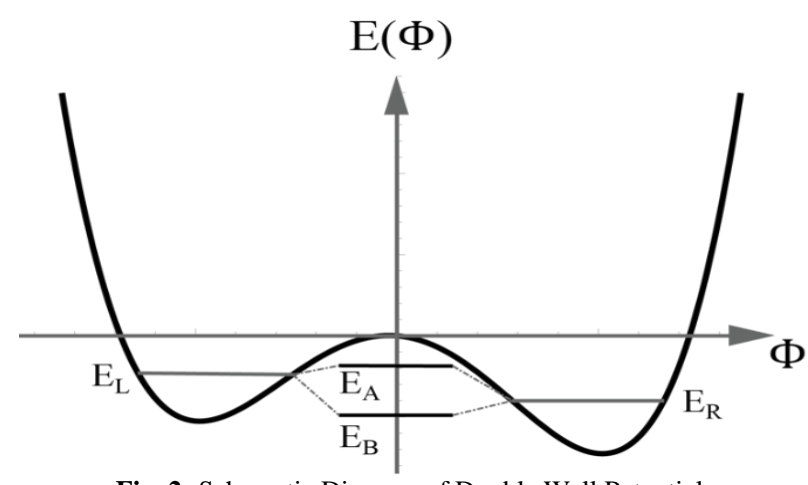

Fig. 2: Schematic Diagram of Double Well Potential.

b) Classical SQUID-based artificial neurons

Based on the two-state transition scheme for generating the sigmoid function to artificial neurons, we analyzed an rf SQUID as a superconducting artificial neuron, which consists of a superconducting loop with the inductance $\mathrm{L}$ interrupted by a Josephson junction as shown in Fig. 3 (a).

The potential energy of rf-SQUID is given by

$$
\mathrm{E}=\mathrm{E}_{\mathrm{L}}\left(\widehat{\Phi}-\widehat{\Phi}_{\mathrm{ex}}\right)^{2}+\mathrm{E}_{\mathrm{J}}\{1-\cos (2 \pi \widehat{\Phi})\}
$$

where $\widehat{\Phi}$ and $\widehat{\Phi}_{\mathrm{ex}}$ are magnetic flux through the superconducting ring and an externally applied magnetic flux normalized by the quantum unit of magnetic flux $\Phi_{0}=\mathrm{h} / 2 \mathrm{e}$ with $\mathrm{h}$ and e being Planck's constant and an elementary electric charge, respectively. The first term is magnetic energy accumulated in the loop with $\mathrm{E}_{\mathrm{L}^{-}}$ $=\Phi_{0}^{2} / 2 \mathrm{~L}$. The second term expresses Josephson coupling energy given by $\mathrm{E}_{\mathrm{J}}=\hbar \mathrm{I}_{\mathrm{C} 0} / 2 \mathrm{e}$ with $\mathrm{I}_{\mathrm{C} 0}$ being the Josephson critical current. Fig. 3 (b) shows the potential profile as a function of $\widehat{\Phi}$ with different applied magnetic flux values $\widehat{\Phi}_{\mathrm{ex}}$. The lowest two minima form a double well potential required to generate the sigmoid function.

The energy difference $\Delta \mathrm{E}$ in (1) is given as

$$
\Delta \mathrm{E}\left(\widehat{\Phi}_{\mathrm{ex}}\right) \cong \mathrm{E}_{\mathrm{L}}\left[\frac{4 \pi^{2} \alpha}{1+2 \pi^{2} \alpha} \widehat{\Phi}_{\mathrm{ex}}+\text { const. }\right]
$$

Where $\alpha=E_{\mathrm{J}} / \mathrm{E}_{\mathrm{L}}$. Therefore, the RF SQUID generates a sigmoid function, where the external magnetic field as the integrated signal is an input determining the activity. (a)

(b)

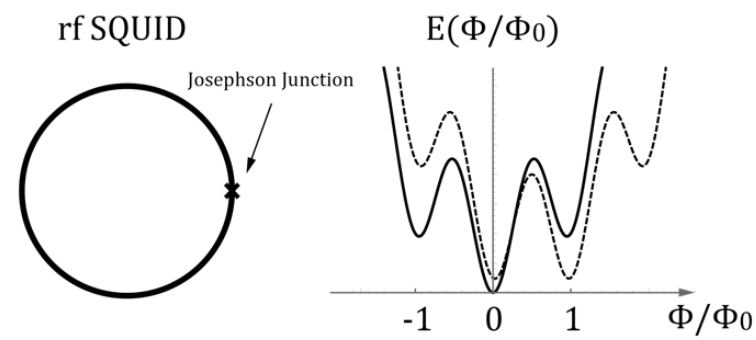

Fig. 2: Schematic Diagram of (A) an Rf SQUID Comprising of A Superconducting Loop Interrupted by A Josephson Junction and (B) Its Potential Profile as A Function of Magnetic Flux Threading the Rf-SQUID Loop with $\widehat{\Phi}_{\mathrm{Ex}}=0$ (Solid Line) and with $\widehat{\Phi}_{\mathrm{Ex}}=0.5$ (Dotted Line).

\section{Quantum squid-based artificial neurons}

a) Quantum rf SQUID

Now let us consider a superconducting artificial neuron working at the quantum-mechanical domain, i.e., $\mathrm{E}_{\mathrm{J}} \leq \mathrm{E}_{\mathrm{c}}$ where $\mathrm{E}_{\mathrm{c}}=$ $4 \mathrm{e}^{2} / 2 \mathrm{C}$. A conventional rf SQUID $\left(\mathrm{E}_{\mathrm{J}}>\mathrm{E}_{\mathrm{c}}\right)$ can be described by a classical particle moving in the potential in (2). Here, the junction capacitance $\mathrm{C}$ corresponds to the mass of the particle. Therefore, when the Josephson junctions are reduced by nanotechnology, the junction capacitance, equivalently the mass, becomes smaller, and as a result, the junctions behave quantum-mechanically. In the case of $E_{J} \leq E_{c}$, the rf SQUID behaves like a quantum-mechanical particle. As is well-known, mechanical variables are replaced by the corresponding operators in quantum mechanics, and commutation relations hold between conjugate operators, i.e., $[\theta, n]=$ i or $[\Phi, Q]=i \hbar$ where $Q$ and $n$ are the electric charge at the junction and the number of Cooper pair difference across the junction, respectively.

In the case that the barrier between wells in the potential (2) is much smaller than the Josephson coupling energy, the rf SQUID potential (2) can be approximated by the biased double well potential as

$$
\mathrm{E}=\mathrm{E}_{\mathrm{L}}\left(\widehat{\Phi}-\widehat{\Phi}_{\mathrm{ex}}\right)^{2}+\mathrm{E}_{\mathrm{J}}\{1-\cos (2 \pi \widehat{\Phi})\}
$$

Where $\phi=\Phi^{\wedge}-0.5$ and $\mathrm{f}=\Phi^{\wedge} \_\mathrm{ex}-0.5$. As a further approximation, the whole Hilbert space of the full Hamiltonian can be mapped on to the subspace spanned by two levels. The resulting Hamiltonian is expressed as

$H=-\frac{1}{2}\left(\epsilon \sigma_{z}+\Delta \sigma_{x}\right)$

where $\epsilon=\mathrm{E} \_$L-E_R is the energy difference between energy levels in each well as shown in Fig. 2 and $\Delta$ stands for tunneling splitting. This Hamiltonian is nothing but qubit Hamiltonian that is a building block of quantum computer in quantum information science.

\section{b) Sigmoid function in quantum rf SQUID}

Now let us consider the sigmoid function required for artificial neurons in quantum rf SQUID. In the classical situation, the sigmoid function was originated from the two-state transition processes between states in double well potential. In quantum mechanical situations as well, the origin is assumed to be same as in the classical one. The probability of finding a particle in the left well of double well potential, $p_{L}$, is given by the coefficient of the left state $|L\rangle$ of the antibonding state $|A\rangle$

$|A\rangle=\sqrt{p_{L}}|L\rangle-\sqrt{p_{R}}|R\rangle$

obtained by diagonalizing the qubit Hamiltonian (5) using unitary transformation 
$\widetilde{H}=D(\beta / 2) H D(\beta / 2)^{\dagger}$

And

$D(\beta / 2)=\exp \left[-i \frac{\beta}{2} \sigma_{y}\right]$

with $\beta=\tan ^{-1}(\Delta / \epsilon)$. The energy spectrum of this diagonalized Hamiltonian is thus represented as

$$
E_{B, A}=\mp \frac{1}{2} \sqrt{\epsilon^{2}+\Delta^{2}}
$$

As shown in Fig. 2. Here, $B(A)$ stands for bonding (antibonding). The bonding state is expressed as

$|A\rangle=D(\beta / 2)^{\dagger}|L\rangle=\cos (\beta / 2)|L\rangle-\sin (\beta / 2)|R\rangle$

As a result, the desired probability is

$$
p_{L}=\cos ^{2}(\beta / 2)=\frac{1}{2}\left[1+\frac{\epsilon}{\sqrt{\epsilon^{2}+\Delta^{2}}}\right] \text {. }
$$

This can be approximated as

$$
p_{L} \cong \frac{1}{1+e^{-\epsilon / 2 \Delta}}
$$

Within the second order of $\epsilon / \Delta$. Therefore, quantum rf SQUID also serves as an artificial neuron with the sigmoid function.

So far, we have discussed the sigmoid function generation of $\mathrm{rf}$ SQUID operating in the quantum mechanical domain based on coherent quantum transition. In the case of incoherent quantum transition, the probability in (11) is reduced to

$p_{L} \cong \frac{1}{1+e^{-\epsilon / 2 \Delta}} \approx 1-e^{-\epsilon / 2 \Delta}$

This has been already observed in early stage of qubit experiment [12].

\section{Conclusion}

We have presented an artificial neuron using rf SQUID working at the quantum-mechanical domain, i.e., quantum rf SQUID. We have showed that quantum rf SQUID serves as an artificial neuron with the sigmoid function based on the model of the coherent quantum transition between quantum states in double well potential. Quantum rf SQUID is nothing but flux qubit that is a building block of quantum computer. Therefore, it might be applicable to artificial neural networks in quantum-mechanical domain, so-called quantum neural networks.

\section{References}

[1] F. Rosenblatt, Principles of Neurodynamics: Perceptrons and the Theory of Brain Mechanisms (Spartan Books, Washington, 1962).

[2] D. E. Rumelhart, G. E. Hinton, and R. J. Williams, "Learning representations by back-propagating errors," Nature, vol. 323, pp. 533 536, October 1986

[3] Sperduti and A. Starita, "Speed up learning and network optimization with extended back propagation," Neural Networks, vol. 6, pp. 365-383, 1993

[4] Y. Harada and E. Goto, "Artificial neural network circuits with Josephson devices," IEEE Transactions on Magnetics, vol. 27, pp. 2863-2866, March 1991.

[5] M. Hidaka and L. A. Akers, "An artificial neural cell implemented with superconducting circuits," Superconductor Science and Technology, vol. 4, pp. 654-657, November 1991.

[6] Y. Mizugaki, K. Nakajima, Y. Sawada, and T. Yamashita, "Superconducting neural circuits using fluxon pulses," Applied Physics Letters, vol. 62, pp. 762-764, February 1993.

[7] Y. Mizugaki, K. Nakajima, Y. Sawada, and T. Yamashita, "Implementation of new superconducting neural circuits using coupled squids," IEEE Transactions on Applied Superconductivity, vol. 4 pp. 1-8, May 1994

[8] T. V. Filippov, Y. A. Polyakov, V. K. Semenov, and K. K. Likharev, "Signal resolution of RSFQ comparators," IEEE Transactions on Applied Superconductivity, vol. 5, pp. 2240-2243, June 1995.

[9] Y. Yamanashi, K. Umeda, and N. Yoshikawa, "Pseudo sigmoid function generator for a superconductive neural network," IEEE Trans. on Applied Superconductivity, vol. 23, pp. 1701004, June 2003.

[10] E. Schegolev, N. V. Klenov, I. I. Soloviev, and M. V. Tereshonok, "Adiabatic superconducting cells for ultra-low-power artificial neural networks,” Beilstein J Nanotechnol, vol. 7, pp. 1397-1403, October 2016.Submitted to publication.

[11] C.H. van der Wal, A.C.J. ter Haar, F. Wilhelm, R.N. Schouten, C.J.P.M. Harmans, T.P. Orlando, S. Lloyd, and J.E. Mooij, "Quantum superposition of macroscopic persistent-current states", Science, vol. 290, pp. 773-777, October 2000 . 